\title{
Modified Norris-Landzberg Model and Optimum Design of Temperature Cycling ALT
}

\author{
F. Q. Sun, ${ }^{a, b, 1}$ J. C. Liu, ${ }^{a, b}$ Z. Q. Cao, ${ }^{b}$ X. Y. Li, ${ }^{a, b}$ and T. M. Jiang ${ }^{b}$ \\ a Science and Technology on Reliability and Environmental Engineering Laboratory, Beihang \\ University, Beijing, China \\ ${ }^{\mathrm{b}}$ School of Reliability and Systems Engineering, Beihang University, Beijing, China \\ ${ }^{1}$ sunfuqiang@buaa.edu.cn
}

Accelerated life testing (ALT) is an effective way to assess the lifetime of a product. Due to the complex nature of its testing profile, it is difficult to carry out temperature cycling ALT. This paper establishes a modified Norris-Landzberg model as acceleration model, and proposes the optimum design method of temperature cycling ALT. First, the FEA method is used to study the influence of temperature cycling profile parameters on the thermal fatigue life of $63 \mathrm{Sn}-37 \mathrm{~Pb}$ solder joints. Then, a modified Norris-Landzberg model is proposed by introducing ramp time and dwell time with an added weight value. Finally, the temperature cycling ALT is regarded as a special multi-stress ALT to study its optimum design method. The uniform design theory is used to determine the combined mode. The optimum model is established with the objective of minimizing the asymptotic variance of the estimation of median lifetime under normal use conditions, and the simulation example shows the workability of the proposed method.

Keywords: temperature cycling ALT, 63Sn-37Pb, Norris-Landzberg model, optimum design, uniform design.

Introduction. Accelerated life testing(ALT) is a test process that subjects a product to conditions that are excess of its normal usage. ALT allows for the collection of extensive failure data over a short time period that can be used to extrapolate and predict lifetime and reliability of products under normal conditions. Currently, ALT technology is an effective way to predict the life expectancy of product, and has been widely recognized in both academia and industry [1]. However, most of previous ALT research has focused on "constant" stress types, such as high-temperature and voltage. It is still unclear how to apply alternating stress types, such as temperature cycling, during ALT.

In practice, many products are mainly subjected to alternating stress during service processing. For example, most on-orbit satellite devices are subject to the temperature cycling stress because of the alternating effects of light and shadow areas. The service environment of aircraft components can be seen as a temperature cycling environment, which is composed of ground parking - takeoff (low tropospheric temperature) - smooth flight (high temperature environment caused by aerodynamic heating) - and landing (low tropospheric temperature). In the process of temperature cycling, the differing thermal expansion coefficients of different materials could lead to thermal fatigue failure. Therefore, it is not effective to only conduct ALT at constant high temperature stress due to different failure mechanisms, and temperature cycling stress should be used in ALT for the products whose main failure mode is thermal fatigue.

Very little research has been conducted on temperature cycling in ALT. Cui [2] carried out a temperature cycle accelerated reliability testing for electronic device packaging, and the Coffin-Manson model and Weibull analysis were used to determine the activation energy related to the crack failure mechanism, which could be used to estimate product reliability under different application conditions. Li et al. [3] applied the temperature cycling ALT to electronic products, and determined the accelerated factor according to data 
derived from similar products. Zhao et al. [4] proposed a data processing approach for ALT under thermal cycling stress based on a modified Coffin-Manson model. However, the previous studies all assume that some parameters of temperature cycling stress profile is constant, and the ALTs are carried out by increasing temperature ranges without any optimum design of test conditions.

This paper studies the optimum design method of the temperature cycling stress ALT program on the basis of the sensitivity analysis of temperature cycling testing profile parameters to the thermal fatigue life. In allusion to the multi-parameters characteristic of the temperature cycling stress profile, the design of experiment (DoE) method is utilized to study the optimum design of the multi-factors and multi-levels temperature cycling ALT.

\section{Temperature Cycling Acceleration Model and Its Improvement.}

1.1. Temperature Cycling Acceleration Model. The relationship between the life characteristics ( $P$ quantile life, median life, mean life) of the product and the stress level is known as the acceleration model, also called the acceleration equation. The acceleration model quantifies the manner in which the life characteristics of the product changes with different stress levels, which is the basis of the life extrapolation in ALT. Thermal fatigue failure caused by temperature cycling stress is a kind of low-cycle fatigue failure, and the Coffin-Manson model is usually used to describe the relationship between temperature range $\Delta T$ and fatigue life,

$$
(\Delta T)^{m} N_{f}=\text { const. }
$$

The Coffin-Manson model only considers the influence of temperature range, while the Norris-Landzberg model (or modified Coffin-Manson model) also takes into account the influence of other temperature cycling profile parameters on the thermal fatigue life, and achieves better results [5]. The expression form of Norris-Landzberg model is

$$
N=\frac{\delta}{(\Delta T)^{\beta_{1}}} \frac{1}{f^{\beta_{2}}} \exp \left(\frac{E_{a}}{K T_{\max }}\right),
$$

where $N$ is fatigue life, $\Delta T$ is the temperature range, $f$ is frequency, $T_{\max }$ is the temperature of the high-temperature dwell stage, $E_{a}$ is the activation energy, $K$ is the Boltzmann constant, and $\delta, \beta_{1}$, and $\beta_{2}$ are undetermined parameters.

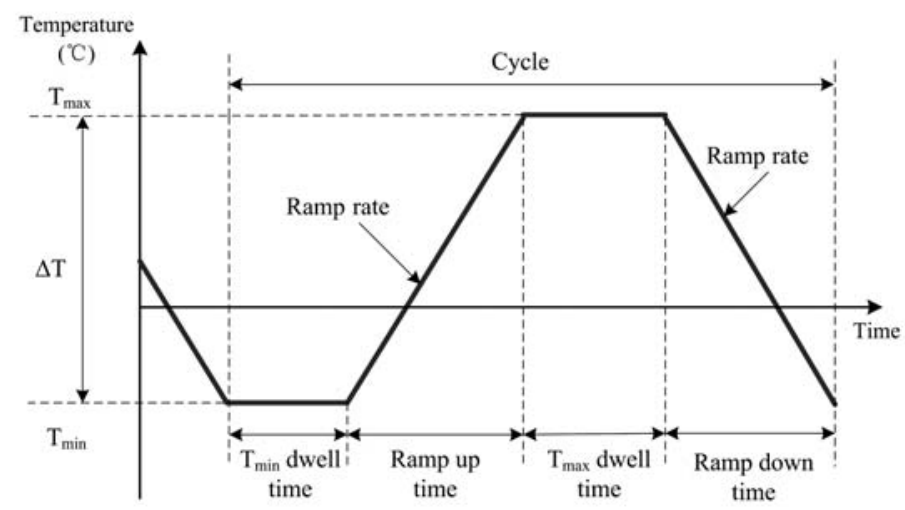

Fig. 1. Temperature cycling profile.

The Norris-Landzberg model is most widely used in temperature cycling testing. The model uses frequency to express the ramp and the dwell stages of the testing profile. A cycle of the temperature cycling profile consists of four stages (Fig. 1), but the Norris- 
Landzberg model characterizes the four stages using a single parameter, and the use of a specific expression for the four time stages instead of the frequency improves the model. Pan et al. [6] uses high temperature dwell time instead of frequency in the NorrisLandzberg model. Dauksher [7] also considers the effect of the high temperature stage on the model and uses the sum of the ramp up time and high-temperature dwell time instead of the frequency. Salmela [8] considers the impact of the whole dwell time, and adds a compensation term of time to the original Norris-Landzberg formula. However, these modified models are used to predict some experimental data very well, but are not universal for all packages [9].

1.2. Sensitivity Analysis of the Model Parameters. In order to improve the NorrisLandzberg model so that it works better with the temperature cycling profile parameters, it is important understand the influence of these various parameters on thermal fatigue life.

The temperature cycling profile can be divided into four stages: ramp up stage, ramp down stage, high-temperature dwell stage and low-temperature dwell stage (Fig. 1). Some researchers use the finite element analysis (FEA) method to study the impact of the temperature cycling profile parameters on thermal fatigue life. Huang et al. [10] combined FEA with ALT in studying the acceleration models of a wafer-level chip-scale package (WLCSP) under thermal cycling conditions, and found that Norris-Landzberg model is more appropriate for WLCSP and the maximum value of the cyclic temperature has significant impact on fatigue life prediction of the package. Zhai et al. [11] studied the effects of the ramp rate and dwell time using laboratory studies and FEA that incorporated stress/strain and plastic work history. Both the FEA modeling and laboratory experiments demonstrated that solder joint fatigue life is more sensitive to dwell time than ramp rate during thermal cycling. Arfaei et al. [12] compared the effects of the four stages using FEA, and found that the influence of the ramp down stage on thermal fatigue is the largest. The traditional view is that the high-temperature stage contributes more to thermal fatigue, but some studies on plastic strain energy found that the most damaging portion of the thermal cycle is the low-temperature dwell stage [13]. However, there is not a unanimous conclusion on the sensitivity of four stages to thermal fatigue.

Therefore, sensitivity analysis for the influence of time parameters of temperature cycling profile on thermal fatigue is conducted. The details of testing profiles are shown in Table 1, with five cycles per profile.

The test objects in this study are $63 \mathrm{Sn}-37 \mathrm{~Pb}$ solder joints of CBGA package. To simplify the calculation, the FEA model consists of a PCB board, a ceramic plate and 16 solder joints. The meshed finite element model is as shown in Fig. 2, and the FEA constraint condition is a fixed underside. The plastic strain amplitude serves as the characterization of thermal fatigue life under temperature cycling stress in FEA. The plastic strain of the model undergoing the No. 3 testing profile is shown in Fig. 3. The place where the outermost solder joint comes in contact with the ceramic plate has the maximum plastic strain amplitude (Fig. 4). Figure 5 describes the plastic strain amplitudes curve changing with time at the contact point between the outermost solder join and the ceramic plate.

The plastic strain amplitudes of 19 simulation tests are shown in Fig. 6. According to the simulation results, the following conclusions can be drawn:

(i) the ramp rate has a significant impact on the plastic strain amplitude. However, when the ramp rate becomes large, the sensitivity of plastic strain amplitude for the ramp rate declines. Nevertheless, changing the ramp rate is still the most effective way to improve the efficiency of the temperature cycling testing;

(ii) the dwell time has a strong impact on the plastic strain amplitude within a small range, but the effect reduces when the dwell time surpasses this range. In addition, the plastic strain amplitude is more sensitive to low-temperature dwell time than high-temperature dwell time. Therefore, dwell time can be appropriately increased when carrying out temperature cycling testing. 
T a b 1 e 1

\section{Temperature Cycling Testing Profiles}

\begin{tabular}{||c|c|c|c|c|c|c|c|c||}
\hline \hline No. & $\begin{array}{c}T_{\max }, \\
{ }^{\circ} \mathrm{C}\end{array}$ & $\begin{array}{c}T_{\min }, \\
{ }^{\circ} \mathrm{C}\end{array}$ & $\begin{array}{c}\text { Ramp } \\
\text { up time } \\
(\min )\end{array}$ & $\begin{array}{c}T_{\max } \\
\text { dwell time } \\
(\min )\end{array}$ & $\begin{array}{c}\text { Ramp } \\
\text { down time } \\
(\min )\end{array}$ & $\begin{array}{c}T_{\min } \\
\text { dwell time } \\
(\mathrm{min})\end{array}$ & $\begin{array}{c}\text { Ramp } \\
\text { up rate } \\
\left({ }^{\circ} \mathrm{C} / \mathrm{min}\right)\end{array}$ & $\begin{array}{c}\text { Ramp } \\
\text { down rate } \\
\left({ }^{\circ} \mathrm{C} / \mathrm{min}\right)\end{array}$ \\
\hline 1 & -55 & 125 & 3 & 25 & 9 & 25 & 60 & 20 \\
\hline 2 & -55 & 125 & 6 & 25 & 9 & 25 & 30 & 20 \\
\hline 3 & -55 & 125 & 9 & 25 & 9 & 25 & 20 & 20 \\
\hline 4 & -55 & 125 & 12 & 25 & 9 & 25 & 15 & 20 \\
\hline 5 & -55 & 125 & 15 & 25 & 9 & 25 & 12 & 20 \\
\hline 6 & -55 & 125 & 9 & 25 & 3 & 25 & 20 & 60 \\
\hline 7 & -55 & 125 & 9 & 25 & 6 & 25 & 20 & 30 \\
\hline 8 & -55 & 125 & 9 & 25 & 12 & 25 & 20 & 15 \\
\hline 9 & -55 & 125 & 9 & 25 & 15 & 25 & 20 & 12 \\
\hline 10 & -55 & 125 & 9 & 40 & 9 & 25 & 20 & 20 \\
\hline 11 & -55 & 125 & 9 & 30 & 9 & 25 & 20 & 20 \\
\hline 12 & -55 & 125 & 9 & 20 & 9 & 25 & 20 & 20 \\
\hline 13 & -55 & 125 & 9 & 10 & 9 & 25 & 20 & 20 \\
\hline 14 & -55 & 125 & 9 & 5 & 9 & 25 & 20 & 20 \\
\hline 15 & -55 & 125 & 9 & 25 & 9 & 40 & 20 & 20 \\
\hline 16 & -55 & 125 & 9 & 25 & 9 & 30 & 20 & 20 \\
\hline 17 & -55 & 125 & 9 & 25 & 9 & 20 & 20 & 20 \\
\hline 18 & -55 & 125 & 9 & 25 & 9 & 10 & 20 & 20 \\
\hline 19 & -55 & 125 & 9 & 25 & 9 & 5 & 20 & 20 \\
\hline \hline
\end{tabular}

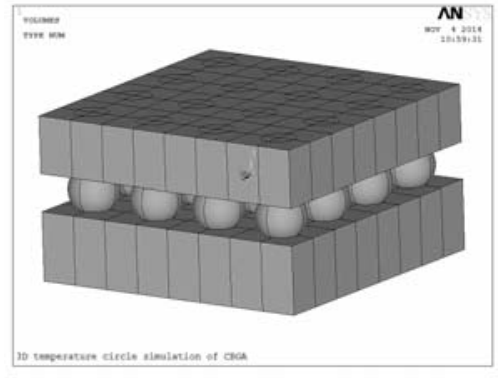

Fig. 2. FEA model.

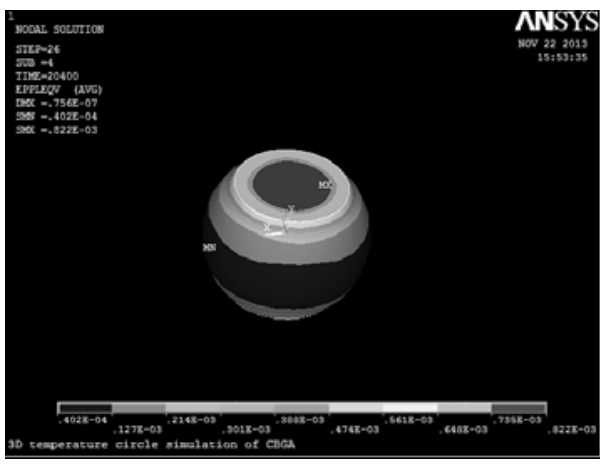

Fig. 4. Plastic strain nephogram of a solder.

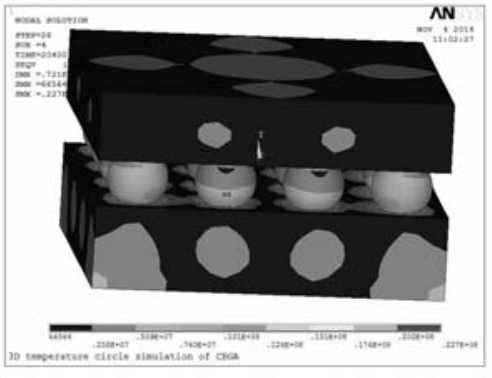

Fig. 3. Plastic strain nephogram.

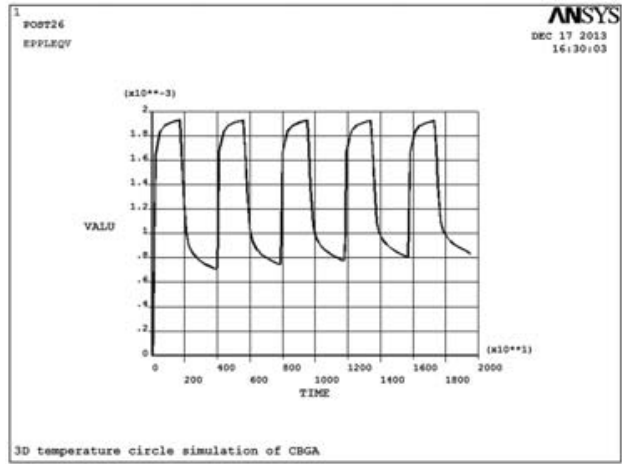

Fig. 5. Plastic strain curve. 

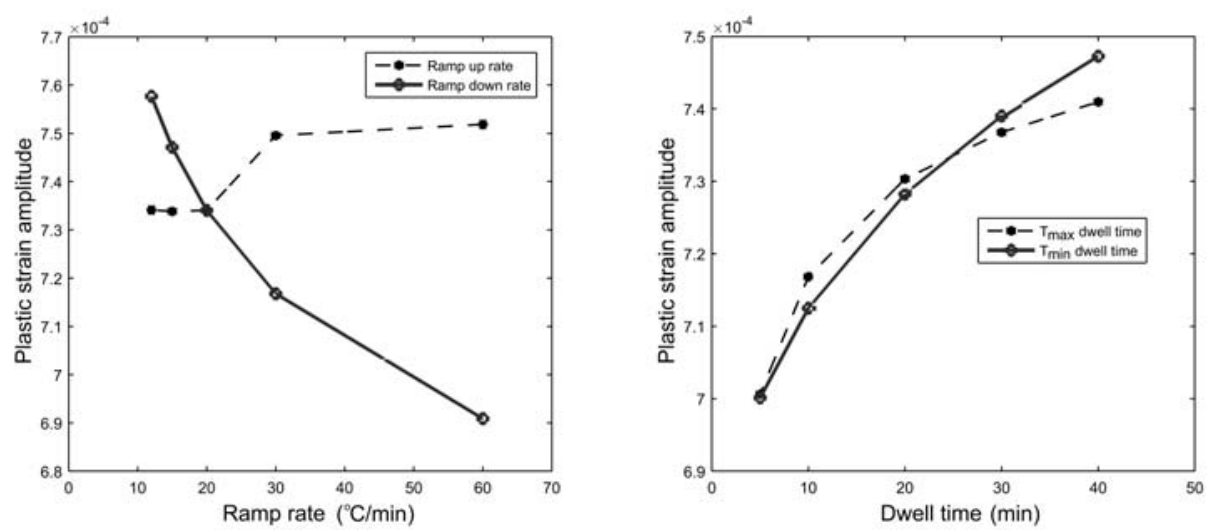

Fig. 6. Plastic strain amplitude curve with ramp rate and dwell time.

1.3. Modified Norris-Landzberg Model. According to the conclusions of the FEA, the ramp rate is more sensitive to fatigue life than the dwell time. Therefore, the modified model should consider the differing influence on the life calculation of these factors. In this paper, the ramp rate and dwell time are introduced into the Norris-Landzberg model, and a weight value is added to describe the different influences of ramp rate and dwell time. The modified model is as follows:

$$
N=\frac{\delta}{(\Delta T)^{a}}\left(t_{1}+p t_{2}\right)^{b} \exp \left(\frac{E_{a}}{k T_{\max }}\right),
$$

where the frequency $f$ in the original Norris-Landzberg model is replaced by $t_{1}+p t_{2}$, and $p$ is the weight value. Because most testing profiles in practice are symmetrical form, meaning the ramp up rate is the same as the ramp down rate, and the high-temperature time is equal to the low-temperature dwell time, and the avoiding model is too complex, $t_{1}$ is the total dwell time, $t_{2}$ is the total ramp time.

In order to verify the accuracy of the modified Norris-Landzberg models, the thermal cycle tests data from [14] (shown in Table 2) is used to calculate fatigue life. The same five CSP packages are tested under every condition. After five CSPs have failed, the life under this condition is defined as the mean failure time.

T a b 1 e 2

\section{Temperature Cycling Test Data}

\begin{tabular}{|c|c|c|c|c|c||}
\hline No. & $\Delta T,{ }^{\circ} \mathrm{C}$ & $\begin{array}{c}f, \\
\text { cycles } / \mathrm{h}\end{array}$ & $\begin{array}{c}\text { Dwell time } \\
(\mathrm{min})\end{array}$ & $\begin{array}{c}\text { Ramp time } \\
(\mathrm{min})\end{array}$ & $\begin{array}{c}\text { Life } \\
(\mathrm{cycles})\end{array}$ \\
\hline 1 & $-40-80$ & 2 & 10 & 5 & 225 \\
\hline 2 & $-40-80$ & 3 & 5 & 5 & 308 \\
\hline 3 & $-40-100$ & 2 & 9 & 6 & 142 \\
\hline 4 & $-40-120$ & 2 & 8 & 7 & 108 \\
\hline 5 & $-20-100$ & 2 & 10 & 5 & 169 \\
\hline 6 & $0-120$ & 2 & 9 & 6 & 131 \\
\hline
\end{tabular}

The parameters values of the modified Norris-Landzberg model are estimated using the testing data of the first five groups, 


$$
N=\frac{465.1221}{(\Delta T)^{0.4449}}\left(t_{1}+2 t_{2}\right)^{-1.1714} \exp \left(\frac{1727}{T_{\max }}\right) \text {, }
$$

where the weight value $p$ is set to 2 .

The test data of group 6 in Table 2 is used to predict the life under $0-120^{\circ} \mathrm{C}$, and the results are shown in Table 3. As can be seen from Table 3, the proposed model has yielded quality results.

$\mathrm{T}$ a b 1 e 3

Calculation Results of Different Models

\begin{tabular}{||c|c|c|}
\hline Model & Predicted life (cycles) & Relative error (\%) \\
\hline The proposed model & 207.755 & 58.59 \\
\hline Original Norris-Landzberg & 219.753 & 67.75 \\
\hline Pan et al. [6] & 231.642 & 76.83 \\
\hline Dauksher [7] & 219.846 & 67.82 \\
\hline Salmela [8] & 483.476 & 269.07 \\
\hline
\end{tabular}

2. Optimum Design of Temperature Cycling ALT. The profile of temperature cycling ALT involves more than 5 parameters, including temperature range, $T_{\max }$, ramp time, dwell time, and cycle frequency. Therefore, it is necessary to make an optimum design of the testing program and search for a reasonable combined mode of the multi-parameters of temperature cycling profile and its multi-levels in order to decrease testing groups and shorten testing time under the premise that the lifetime estimation accuracy remains unchanged.

The uniform design DoE theory [15] is used to determine the combined mode of the multi-parameter and multiple levels of the temperature cycling ALT profile. Under the constraint of the testing boundary conditions (such as the maximum accelerated stress levels that do not change the failure mechanism of the product), the optimum problem is established with the objective of minimizing asymptotic variance in the estimation of $P$ quantile life, and optimal testing variables including stress levels and sample size are given.

2.1. The Combined Mode of Multi-Parameters of Temperature Cycling ALT. According to Eq. (2), the Norris-Landzberg model contains three temperature cycling profile parameters, temperature range, cycle frequency and high temperature $T_{\max }$. Increasing the value of each of these parameters could be conducive for enhancing the damage effect of temperature cycling and create acceleration. If the three parameters are all accelerated, and each parameter has three levels, there would be $3^{3}=27$ combinations of stress levels, which is too complex. Therefore, in order to make the optimum ALT program and achieve the most testing efficiency, the key question is how to determine the optimal combination mode of the multi-parameters and multi-levels.

The three parameters of the temperature cycling profile, temperature range, cycle frequency, and high temperature $T_{\max }$, are treated as three different kinds of stresses. The uniform design theory is used to determine the combined mode of different stresses. According to Uniform design theory, testing times is equal to the number of stress levels, so the testing times can be greatly reduced under the premise of guaranteed estimation accuracy. For example, if a testing has three stresses and four levels, there are $4^{3}=64$ kinds of stress combinations. According to uniform design table $U_{4}\left(4^{3}\right)$ [16], only four groups need to be implemented. These stress combinations are shown in Table 4. 
$\mathrm{T}$ a b 1 e 4

\section{$U_{4}\left(4^{3}\right)$ Uniform Design Table}

\begin{tabular}{|c|c|c|c|}
\hline Testing number & Stress factor A & Stress factor B & Stress factor C \\
\hline 1 & 1 & 3 & 1 \\
\hline 2 & 2 & 1 & 4 \\
\hline 3 & 3 & 4 & 3 \\
\hline 4 & 4 & 2 & 2 \\
\hline
\end{tabular}

\subsection{Reliability Statistical Model.}

2.2.1. Model Assumption. The thermal fatigue life of electronic products under temperature cycling stress usually follows the Weibull distribution. We use the Weibull distribution to make statistical analyses for time censored temperature cycling ALT, and the model assumption is as follows:

Assumption I: For any stress level, either normal operation stress level or accelerated stress level, the life of the product follows the Weibull distribution. The probability density function are

$$
f(t)=\frac{m}{\eta}\left(\frac{t}{\eta}\right)^{m-1} \exp \left[-\left(\frac{t}{\eta}\right)^{m}\right],
$$

where $m$ is the shape parameter and $\eta$ is the characteristic life. This assumption implies that changes in stress level cannot change the type of the life distribution.

Assumption II: The shape parameter $m$ the Weibull distribution stays unchanged under different stress levels, meaning changes in stress level do not change the failure mechanism of the product.

Assumption III: The accelerated model between the characteristic life $\eta$ and the applied stress level $S$ is written as

$$
\ln \eta=a+b \varphi(S)
$$

where $a$ and $b$ are undetermined parameters.

A logarithmic transformation is performed for the Norris-Landzberg model in Eq. (2),

$$
\ln \eta=\gamma_{0}+\gamma_{1} S_{1}+\gamma_{2} S_{2}+\gamma_{3} S_{3},
$$

where $\gamma_{0}=\ln \delta, \gamma_{1}=-a, \gamma_{2}=b, \gamma_{3}=c, S_{1}=\ln \Delta T, S_{2}=\ln f, S_{3}=1 / T_{\max }$, and $S_{1}$, $S_{2}$, and $S_{3}$ represent the temperature range, cycle frequency, and high temperature $T_{\max }$, which are three different kinds of accelerated stress.

Let $\theta=\ln t$, and using variable substitution, Eq. (5) can be transformed into the following form:

$$
f(\theta)=\frac{1}{\sigma} \exp \left(\frac{\theta-\mu}{\sigma}\right) \exp \left[-\exp \left(\frac{\theta-\mu}{\sigma}\right)\right],
$$

where $\mu=\ln \eta=\gamma_{0}+\gamma_{1} S_{1}+\gamma_{2} S_{2}+\gamma_{3} S_{3}$ and $\sigma=1 / m$. From this variable substitution, the Weibull distribution is transformed into the extreme value distribution form. This model is a linear-extreme value statistical model, and its expression is more general.

2.2.2. The Likelihood Function and Fisher Information Matrix of Censored Samples. Assume that the total sample size of a time-censored temperature cycling ALT is $n$ and the censoring time is $\tau_{i}$. For the $i$ th specimen, if failures occur at time $t_{i}$, the likelihood function of the general statistical model is 


$$
L_{i}^{\prime}=\ln f\left(\theta_{i}\right)=-\ln \sigma+\left(\theta_{i}-\mu\right) / \sigma-\exp \left[\left(\theta_{i}-\mu\right) / \sigma\right]
$$

where $\theta_{i}=\ln t_{i}$.

If no failures occur before the censoring time $\tau_{i}$, the likelihood function is

$$
L_{i}^{\prime \prime}=\ln R\left(\zeta_{i}\right)=\ln \left[1-F\left(\xi_{i}\right)\right]=-\exp \left[\left(\zeta_{i}-\mu\right) / \sigma\right],
$$

where $\xi_{i}=\ln \tau_{i}$.

If we let $I_{i}=\left\{\begin{array}{ll}1, & \theta_{i} \leq \zeta_{i}, \\ 0, & \theta_{i}>\zeta_{i},\end{array}\right.$ then the likelihood function of the $i$ th specimen is

$$
L_{i}=I_{i} \ln f\left(\theta_{i}\right)+\left(1-I_{i}\right) \ln R\left(\zeta_{i}\right) .
$$

Let $z_{i}=\left(\theta_{i}-\mu\right) / \sigma, \xi_{i}=\left(\xi_{i}-\mu\right) / \sigma$, and the likelihood function of $n$ specimens is

$$
L=\sum_{i=1}^{n} L_{i}=\sum_{i=1}^{n}\left\{I_{i}\left[-\ln \sigma+z_{i}-\exp \left(z_{i}\right)\right]+\left(1-I_{i}\right)\left[-\exp \left(\xi_{i}\right)\right]\right\}
$$

According to MLE theory, $\Sigma$, the variance and covariance matrix of the estimators of model parameters $\hat{\gamma}_{0}, \hat{\gamma}_{1}, \hat{\gamma}_{2}, \hat{\gamma}_{3}, \hat{\sigma}$, is the inverse matrix of Fisher information matrix $F$, meaning $\Sigma=F^{-1}$. It is difficult to calculate the variance and covariance matrix directly, so $\Sigma$ can be obtained from calculating Fisher information matrix $F$ indirectly. $F$ is the mathematical expectation of negative second order partial derivative matrix of the likelihood function,

$$
F=\frac{1}{\sigma^{2}} \sum_{i=1}^{n}\left[\begin{array}{ccccc}
A\left(\xi_{i}\right) & S_{1, i} A\left(\xi_{i}\right) & S_{2, i} A\left(\xi_{i}\right) & S_{3, i} A\left(\xi_{i}\right) & B\left(\xi_{i}\right) \\
S_{1, i} A\left(\xi_{i}\right) & S_{1, i}^{2} A\left(\xi_{i}\right) & S_{1, i} x_{2, i} A\left(\xi_{i}\right) & S_{1, i} x_{3, i} A\left(\xi_{i}\right) & S_{1, i} B\left(\xi_{i}\right) \\
S_{2, i} A\left(\xi_{i}\right) & S_{1, i} S_{2, i} A\left(\xi_{i}\right) & S_{2, i}^{2} A\left(\xi_{i}\right) & S_{2, i} x_{3, i} A\left(\xi_{i}\right) & S_{2, i} B\left(\xi_{i}\right) \\
S_{3, i} A\left(\xi_{i}\right) & S_{1, i} S_{3, i} A\left(\xi_{i}\right) & S_{2, i} S_{3, i} A\left(\xi_{i}\right) & S_{3, i}^{2} A\left(\xi_{i}\right) & S_{3, i} B\left(\xi_{i}\right) \\
B\left(\xi_{i}\right) & S_{1, i} B\left(\xi_{i}\right) & S_{2, i} B\left(\xi_{i}\right) & S_{3, i} B\left(\xi_{i}\right) & C\left(\xi_{i}\right)
\end{array}\right],
$$

where

$$
\begin{gathered}
A\left(\xi_{i}\right)=1-\exp \left[-\exp \left(\xi_{i}\right)\right], \\
B\left(\xi_{i}\right)=\int_{-\infty}^{\xi_{i}} z_{i} \exp \left(2 z_{i}\right) \exp \left[-\exp \left(z_{i}\right)\right] d z_{i}+\xi_{i} \exp \left(\xi_{i}\right) \exp \left[-\exp \left(\xi_{i}\right)\right], \\
C\left(\xi_{i}\right)=\int_{-\infty}^{\xi_{i}}\left[z_{i} \exp \left(z_{i}\right)+\exp \left(z_{i}\right)-1\right] z_{i} \exp \left(z_{i}\right) \exp \left[-\exp \left(z_{i}\right)\right] d z_{i}+ \\
+\left(\xi_{i}+\xi_{i}^{2}\right) \exp \left(\xi_{i}\right) \exp \left[-\exp \left(\xi_{i}\right)\right] .
\end{gathered}
$$

2.2.3. The Asymptotic Variance of the Estimation of Pth Quantile of Product's Lifetime. For the linear-extreme value model, the $P$ th quantile $g_{P}\left(S_{1}, S_{2}, S_{3}\right)$ of the life distribution under stress $S_{1}, S_{2}$, and $S_{3}$ is the function of $\gamma_{0}, \gamma_{1}, \gamma_{2}, \gamma_{3}$, and $\sigma$, and its maximum likelihood estimation is

$$
\hat{g}_{P}\left(S_{1}, S_{2}, S_{3}\right)=\mu+z_{P} \sigma=\hat{\gamma}_{0}+\hat{\gamma}_{1} S_{1}+\hat{\gamma}_{2} S_{2}+\hat{\gamma}_{3} S_{3}+z_{P} \hat{\sigma},
$$


where $z_{P}$ is the $P$ th quantile of the standard extreme value distribution, meaning $z_{P}=$ $=\ln \ln [1 /(1-P)]$. Generally, $P=0.5$, such that $z_{P}=-0.3665$, which is the product's median life.

When $n \rightarrow \infty, g_{P}\left(S_{1}, S_{2}, S_{3}\right)$ follows an asymptotically normal distribution with mean $\hat{g}_{P}\left(S_{1}, S_{2}, S_{3}\right)$, and variance is

$$
\operatorname{var}\left(\hat{g}_{P}\right)=h \Sigma h^{\prime}=h F^{-1} h^{\prime},
$$

where $h=\left(\partial g_{P} / \partial \gamma_{0}, \partial g_{P} / \partial \gamma_{1}, \partial g_{P} / \partial \gamma_{2}, \partial g_{P} / \partial \gamma_{3}, \partial g_{P} / \partial \sigma\right)=\left(1, S_{1}, S_{2}, S_{3}, z_{P}\right)$.

Hence, the asymptotic variance of the estimation of median life under normal use condition is

$$
\begin{gathered}
\operatorname{var}\left(\hat{g}_{0.5}\left(S_{1,0}, S_{2,0}, S_{3,0}\right)\right)= \\
=\left(1, S_{1,0}, S_{2,0}, S_{3,0}, z_{0.5}\right) F^{-1}\left(1, S_{1,0}, S_{2,0}, S_{3,0}, z_{0.5}\right)^{\prime}=\left(\sigma^{2} / n\right) V,
\end{gathered}
$$

where $V$ is called variance factor.

\subsection{Optimal Model.}

2.3.1. Objective Function. The temperature cycling profile has three accelerated parameters, temperature range $\Delta T$, cycle frequency $f$, and high temperature $T_{\text {max }}$, which can be expressed by $S_{1}, S_{2}$, and $S_{3}$. Assuming that each kind of stress has $K$ stress levels, there will be $K^{3}$ combinations of different stress levels. Suppose that the stress level of the $l$ th kind of stress $S_{l}$ under normal use condition is $S_{l, 0}$, the limit stress level is $S_{l, \max }(l=1, \ldots, 3)$, and the failure mechanism of product is unchanged under $S_{l, \max }$. The ALT testing stress level is $S_{l, k}\left(S_{l, 0}<S_{l, k}<S_{l, \max }\right)(k=1, \ldots, K)$. How to select optimal combined mode from $K^{3}$ different combinations and how to choose $S_{l, k}$ among $S_{l, 0}-S_{l, \max }$ are all a part of the optimum design of temperature cycling ALT.

According to uniform design theory, testing times are equal to the number of stress levels, so only four groups of temperature cycling ALT need to be implemented. Suppose that the proportion of sample size of the $i$ th combination of stresses is $p_{i}$, then the Fisher information matrix (13) can be written as

$$
F_{K}=\frac{n}{\sigma^{2}} \sum_{i=1}^{K}\left[\begin{array}{ccccc}
p_{i} A\left(\xi_{i}\right) & p_{i} S_{1, i} A\left(\xi_{i}\right) & p_{i} S_{2, i} A\left(\xi_{i}\right) & p_{i} S_{3, i} A\left(\xi_{i}\right) & p_{i} B\left(\xi_{i}\right) \\
p_{i} S_{1, i} A\left(\xi_{i}\right) & p_{i} S_{1, i}^{2} A\left(\xi_{i}\right) & p_{i} S_{1, i} S_{2, i} A\left(\xi_{i}\right) & p_{i} S_{1, i} S_{3, i} A\left(\xi_{i}\right) & p_{i} S_{1, i} B\left(\xi_{i}\right) \\
p_{i} S_{2, i} A\left(\xi_{i}\right) & p_{i} S_{1, i} S_{2, i} A\left(\xi_{i}\right) & p_{i} S_{2, i}^{2} A\left(\xi_{i}\right) & p_{i} S_{2, i} S_{3, i} A\left(\xi_{i}\right) & p_{i} S_{2, i} B\left(\xi_{i}\right) \\
p_{i} S_{3, i} A\left(\xi_{i}\right) & p_{i} S_{1, i} S_{3, i} A\left(\xi_{i}\right) & p_{i} S_{2, i} S_{3, i} A\left(\xi_{i}\right) & p_{i} S_{3, i}^{2} A\left(\xi_{i}\right) & p_{i} S_{3, i} B\left(\xi_{i}\right) \\
p_{i} B\left(\xi_{i}\right) & p_{i} S_{1, i} B\left(\xi_{i}\right) & p_{i} S_{2, i} B\left(\xi_{i}\right) & p_{i} S_{3, i} B\left(\xi_{i}\right) & p_{i} C\left(\xi_{i}\right)
\end{array}\right] .
$$

According to Eq. (16), the asymptotic variance of the estimator of product median life under normal stress level can be obtained by

$$
\begin{gathered}
\operatorname{var}\left(\hat{g}_{0.5}\left(S_{1,0}, S_{2,0}, S_{3,0}\right)\right)= \\
=\left(1, S_{1,0}, S_{2,0}, S_{3,0}, z_{0.5}\right) F_{K}^{-1}\left(1, S_{1,0}, S_{2,0}, S_{3,0}, z_{0.5}\right)^{\prime}=\left(\sigma^{2} / n\right) V_{K},
\end{gathered}
$$

where $V_{K}$ is variance factor when the number of the stress levels is $K$.

The optimum objective is maximizing the accuracy of the evaluation of the product's median life under normal use conditions, meaning minimizing the asymptotic variance of the estimation of the product's median life under normal stress level. Hence, the variance factor $V_{K}$ is chosen as the objective function for the optimum design of ALT. Therefore, the objective function is 


$$
\min \left(V_{K}\right)=\min \left[\left(n / \sigma^{2}\right) \operatorname{var}\left(\hat{g}_{0.5}\left(S_{1,0}, S_{2,0}, S_{3,0}\right)\right)\right]
$$

2.3.2. Constraint Conditions and Decision Variables. The censoring time $\tau$, maximum testing stress levels $S_{1, K}, S_{2, K}, S_{3, K}$ and normal operational stress levels $S_{1,0}, S_{2,0}$, $S_{3,0}$ should be given before the optimum design of ALT is carried out. Generally, the selected minimum testing stress level is equal to or slightly higher than the normal stress level. If possible, the maximum stress levels $S_{1, K}, S_{2, K}$, and $S_{3, K}$ should be close to the limit stress level if the failure mechanism remains unchanged. That is $S_{1,0}<S_{1,1}<S_{1,2}<\ldots$ $\ldots<S_{l, K} \leq S_{l, \max }(l=1,2, k=1, \ldots, K)$ and $S_{3,0}>S_{3,1}>S_{3,2}>\ldots>S_{3, K} \geq S_{3, \min }$.

Moreover, testing under each combination of stresses should have an acceleration characteristic, meaning the acceleration factor of each combination of stresses should be greater than a certain value,

$$
\alpha_{i \sim 0}=\eta_{0} / \eta_{i}=\exp \left[\gamma_{1}\left(S_{1,0}-S_{1, i}\right)+\gamma_{2}\left(S_{2,0}-S_{2, i}\right)+\gamma_{3}\left(S_{3,0}-S_{3, i}\right)\right]>\alpha_{\min } .
$$

The parameter estimates $\hat{\gamma}_{0}, \hat{\gamma}_{1}, \hat{\gamma}_{2}, \hat{\gamma}_{3}$, and $\hat{\sigma}$ of variance factor $V_{K}$ can be roughly determined according to the designers' engineering experience, similar product data or the results of trial testing. After determining the above parameters, the variance factor $V_{K}$ of the estimation of product's median life will depend on the other $K-1$ stress levels $S_{l, k}$ and the proportion $p_{i}$ of sample size of each combination of stresses. Therefore, the decision variables are $S_{l, k}(l=1,2,3, k=1, \ldots, K-1)$ and $p_{i}(i=1, \ldots$, $K-1$ ), where $S_{1}=\ln \Delta T, S_{2}=\ln f$, and $S_{3}=1 / T_{\max }$.

The optimization problem can be solved as follows:

$$
\begin{aligned}
& \min V_{K} \\
& S_{l, 0}<S_{l, 1}<S_{l, 2}<\ldots<S_{l, K} \leq S_{l, \max }, \quad l=1,2, \\
& S_{3,0}>S_{3,1}>S_{3,2}>\ldots>S_{3, K} \geq S_{3, \min }, \\
& 0<p_{i}<1, \quad \sum_{i=1}^{K-1} p_{i}=1-p_{K}, \quad i=1,2, \ldots, K-1, \\
& \alpha_{i \sim 0}=\exp \left[\gamma_{1}\left(S_{1,0}-S_{1, i}\right)+\gamma_{2}\left(S_{2,0}-S_{2, i}\right)+\gamma_{3}\left(S_{3,0}-S_{3, i}\right)\right]>\alpha_{\text {min }} .
\end{aligned}
$$

According to the actual range constraints of the decision variables, we can build a scheme set for the optimization problem including the solution spaces for these decision variables, and the scheme with minimum $V_{K}$ can be chosen as the optimal solution.

2.4. Simulation Study. The CBGA packages of the $63 \mathrm{Sn}-37 \mathrm{~Pb}$ are used to test the optimum design of the temperature cycling ALT. Based on the product design information and limit stresses analysis, the normal operating conditions are between 0 and $80^{\circ} \mathrm{C}$, and the cycle frequency is 2 cycle $/ \mathrm{h}$. The limit stress level is between -40 and $120^{\circ} \mathrm{C}$, and the cycle frequency is 3 cycle $/ \mathrm{h}$, such that $\Delta T_{0}=80^{\circ} \mathrm{C}, f_{0}=2 \mathrm{cycle} / \mathrm{h}, T_{\max , 0}=80^{\circ} \mathrm{C}, \Delta T_{\max }=$ $=160^{\circ} \mathrm{C}, f_{\max }=3 \mathrm{cycle} / \mathrm{h}$, and $T_{\max , \max }=120^{\circ} \mathrm{C}$. Using the logarithmic transformation according to Eq. (7), such that $S_{1}=\ln \Delta T, S_{2}=\ln f$, and $S_{3}=1 / T_{\max }$, the normal stress levels and limit stress levels of $S_{1}, S_{2}$, and $S_{3}$ can be obtained, $S_{1,0}=4.382$, $S_{2,0}=0.6931, S_{3,0}=0.002832, S_{1, \max }=5.075, S_{2, \max }=1.099, S_{3, \min }=0.0025$.

The stress applied method of this time-censored temperature cycling ALT is constant stress, and there are four stress levels. According to the uniform design table $U_{4}\left(4^{3}\right)$, only four stress combinations need to be implemented, and the sample size of each group is same, meaning $p_{i}=1 / 4$. The censoring time $\tau$ can be determined according to trial testing, and here $\tau=350$ cycles. The minimum acceleration factor value is $\alpha_{\min }=1$. The rough estimates of reliability statistical model parameters are 


$$
\hat{\gamma}_{0}=9.517, \quad \hat{\gamma}_{1}=-2.064, \quad \hat{\gamma}_{2}=0.345, \quad \hat{\gamma}_{3}=2006.4, \quad \hat{\sigma}=0.92 .
$$

The optimum problem is then established with the objective of minimizing the variance factor $V_{K}$ of the estimation of product's median life under normal stress levels. Optimal testing variables $S_{l, k}(l=1,2,3, k=1, \ldots, K-1)$ are given, as shown in Table 5, and the optimal objective function $V_{K}=443.871$. The optimal temperature cycling ALT scheme is given according to the $U_{4}\left(4^{3}\right)$ uniform design table, as shown in Table 6 .

$\mathrm{T}$ a b 1 e 5

\section{Optimal Decision Variables}

\begin{tabular}{|c|c|c|c|c|c||}
\hline$S_{1}$ & $\Delta T,{ }^{\circ} \mathrm{C}$ & $S_{2}$ & $f$, cycle/h & $S_{3}$ & $T_{\max },{ }^{\circ} \mathrm{C}$ \\
\hline 4.4495 & 86 & 0.6941 & 2.0 & 0.00282 & 81 \\
\hline 4.6580 & 105 & 0.8291 & 2.3 & 0.00272 & 94 \\
\hline 4.8665 & 130 & 0.9640 & 2.6 & 0.00261 & 110 \\
\hline 5.0750 & 160 & 1.0990 & 3.0 & 0.00250 & 120 \\
\hline
\end{tabular}

$\mathrm{T}$ a b 1 e 6

Optimal Temperature Cycling ALT Scheme

\begin{tabular}{|c|c|c|c||}
\hline Testing groups & $\Delta T,{ }^{\circ} \mathrm{C}$ & $f$, cycle $/ \mathrm{h}$ & $T_{\max },{ }^{\circ} \mathrm{C}$ \\
\hline 1 & 86 & 2.6 & 81 \\
\hline 2 & 105 & 2.0 & 120 \\
\hline 3 & 130 & 3.0 & 110 \\
\hline 4 & 160 & 2.3 & 94 \\
\hline
\end{tabular}

Usually, the accelerated stress levels of ALT are determined through equal distribution among the limit stress level and normal stress level. We assume that other testing parameters of this average allocation ALT scheme are the same as this paper, and the stress combinations, sample proportion, acceleration model and censoring time are all unchanged. The variance factor in this case is $V_{K}=537.755$. As can be seen, it is much higher than the variance factor of optimal testing scheme in this paper.

Conclusions. According to the damage mechanism of temperature cycling stress to electronics, with plastic strain amplitude as the presentation of thermal fatigue life, this paper conducts the finite element thermal simulation analysis, and studies the influence of profile parameters, including dwell time and ramp rate, on the thermal fatigue life of solder joints. According to the conclusions of sensitivity analysis, the Norris-Landzberg model is improved by introducing ramp time and dwell time with an added weight value to describe the effects of cycle frequency. This study lays the foundation for temperature cycling accelerated testing.

Temperature cycling ALT is regarded as a special multi-stress ALT to study its optimum design method. The uniform design method, a type of DoE theory, is used to determine the combined mode of the multi-parameters and multiple levels of the temperature cycling ALT profile. The optimum model of the ALT scheme is established with the objective of minimizing the asymptotic variance of the estimation of $P$ th quantile of product's lifetime under normal stress level, and the optimal testing variables, including stress levels and sample size, are given. The results of the simulation examples suggest that the proposed optimum design method of temperature cycling ALT scheme can reduce testing times and cost. 
Acknowledgments. This study was supported by the National Natural Science Foundation of China (No. 61104182).

1. Ali A. Ismail, "On designing constant-stress partially accelerated life tests under time-censoring," Strength Mater., 46, No. 1, 132-139 (2014).

2. H. Cui, "Accelerated temperature cycle test and Coffin-Manson model for electronic packaging," in: Proc. Annual Reliability and Maintainability Symp. (Jan. 24-27, 2005, Alexandria, VA, USA), IEEE (2005), pp. 556-560.

3. S. Z. Li, X .Y. Li, T. M. Jiang, and J. J. Liu, "Application of temperature cycle based ALT technology in electronic products," Equip. Environ. Eng., 6, No. 6, $73-77$ (2009).

4. S. S. Zhao, Y. X. Chen, and Y. N. Jia, "Design and assessment of accelerated life testing based on modified Coffin-Manson model," Struct. Environ. Eng., 40, No. 4, 52-58 (2013).

5. K. C. Norris and A. H. Landzberg, "Reliability of controlled collapse interconnections," IBM J. Res. Develop., 13, 266-271 (1969).

6. N. Pan, G. A. Henshall, F. Billaut, et al., "An acceleration model for Sn-Ag-Cu solder joint reliability under various thermal cycle conditions," in: Proc. Int. Conf. on SMTA (Sept. 25-29, 2005, Chicago, IL, USA) (2005), pp. 876-883.

7. W. Dauksher, "A second-level SAC solder-joint fatigue-life prediction methodology," IEEE Trans. Device Mater. Reliab., 8, No. 1, 168-173 (2008).

8. O. Salmela, "Acceleration factors for lead-free solder materials," IEEE Trans. Comp. Pack. Technol., 30, No. 4, 700-707 (2007).

9. L. Yang, J. B. Bernstein, and T. Koschmieder, "Assessment of acceleration models used for BGA solder joint reliability studies," Microelectr. Reliab., 49, No. 12, 15471554 (2009).

10. X. H. Huang, W. F. Wu, and P. L. Chou, "Fatigue life and reliability prediction of electronic packages under thermal cycling conditions through FEM analysis and acceleration models," in: Proc. 14th Int. Conf. on Electronic Materials and Packaging (EMAP) (Dec. 13-16, 2012, Hongkong) (2012), pp. 1-6.

11. C. J. Zhai, Sidharth, and R. Blish, II, "Board level solder reliability versus ramp rate and dwell time during temperature cycling," IEEE Trans. Device Mater. Reliab., 3, No. 4, 207-212 (2003).

12. B. Arfaei, S. Mahin-Shirazi, S. Joshi, et al., "Reliability and failure mechanism of solder joints in thermal cycling tests," in: Proc. 63rd on IEEE Electronic Components and Technology Conference (May 28-31, 2013, Las Vegas, NV, USA), IEEE (2013), pp. $976-985$.

13. A. Syed, "Limitations of Norris-Landzberg equation and application of damage accumulation based methodology for estimating acceleration factors for $\mathrm{Pb}$ free solders," Proc. 11th Int. Conf. on Thermal, Mechanical \& Multi-Physics Simulation, and Experiments in Microelectronics and Microsystems (EuroSimE) (Apr. 26-28, 2010, Bordeaux, France), IEEE (2010), pp. 1-11.

14. I. Shohji, H. Mori, and Y. Orii, "Solder joint reliability evaluation of chip scale package using a modified Coffin-Manson equation," Microelectr. Reliab., 44, No. 2, 269-274 (2004).

15. W. H. Chen, H. Y. Feng, P. Qian, et al., "Theory \& method for optimum design of accelerated life test plan under multiple stresses," Chin. J. Mech. Eng., 42, No. 12, 101-105 (2007).

16. http://uic.edu.hk/isci/UniformDesign/UD\%20Tables.html. 